\title{
Eye Deviation during Rotation in Darkness in Trait Anxiety: An Early Expression of Perceptual Avoidance?
}

\author{
Isabelle Viaud-Delmon, Isabelle Siegler, Isabelle Israël, Roland Jouvent, \\ and Alain Berthoz
}

Background: Patients with dizziness and patients with panic disorder and agoraphobia share a common symptomatology. Numerous studies have investigated a potential link between anxiety and the vestibular system, but few of them have addressed the specific topic of spatial representation.

Methods: Passive whole-body rotations in the horizontal plane were imposed on two groups of subjects who differed in their level of trait anxiety. Subjects were seated on a mobile robot in darkness. After each passive rotation, subjects were asked to reproduce the stimulus by driving the robot with a joystick and to perform a rotation of the same magnitude. Eye movements were recorded and analyzed.

Results: No difference in either perception (accuracy in the reproduction task) or in VOR gain was found between the two groups of subjects. Mean eye deviation, caused by fast phases of the nystagmus, differed in the two groups. It was typically in the anticompensatory direction in the non-anxious group, and in the compensatory direction the anxious group. Such compensatory movement may be explained by an egocentric orientation strategy, that may in turn indicate a lack of interest toward the visual surroundings.

Conclusions: An egocentric strategy for self-orientation exhibited at a level below the threshold of awareness could reveal the existence of a physiological mode of processing leading to agoraphobic avoidance. Biol Psychiatry 2000;47:112-118 (C) 2000 Society of Biological Psychiatry

Key Words: Anxiety, eye movements, spatial perception, vestibulo ocular reflex, vestibular nystagmus, humans

From the Laboratoire de Physiologie de la Perception et de l'Action, CNRSCollege de France (IV-D, IS, II, AB) and CNRS UMR 7593, Hôpital de la Salpêtriére (IV-D, RJ), Paris, France.

Address reprint requests to Isabelle Viaud-Delmon, CNRS-College de France, Laboratoire de Physiologie de la Perception et de l'Action, 11 place Marcelin Berthelot, 75005 Paris, France.

Received January 5, 1999; revised March 1, 1999; accepted April 27, 1999.

\section{Introduction}

$\mathrm{C}$ omplaints of dizziness are frequently associated with anxiety (Yardley et al 1998). For this reason, several studies have tried to find a link between panic disorder with or without agoraphobia and vestibular system dysfunction. Yet, even though some vestibular abnormalities have been demonstrated in these patients (Clark et al 1994; Hoffman et al 1994; Jacob et al 1985, 1996; Sklare et al 1990), it is difficult to demonstrate a cause-and-effect relationship between panic disorder and vestibular dysfunction. This does not rule out a possible influence of anxiety on normal vestibular function.

The vestibular system functions in conjunction with other sensory modalities to determine our perception of bodily orientation in space. Furthermore, vestibular induced behavior can also reflect a strategy. Therefore in attempting to make a connection between vestibular function and anxiety, one should look not only for vestibular dysfunction but also for differences in the use of vestibular information, that could be at the origin of different perceptual strategies. Schilder (1933) already hinted in this direction: "The vestibular system is not only a perceptive organ, but also a reflex organ that reacts to movements and to positions of the head in space [ ... ] But the vestibular system is also under the influence of the personality, of the brain function as a whole."

The primary aim of this study was to evaluate the way that the level of trait anxiety can be correlated with vestibular reflex function. Trait anxiety is measured with relatively stable personality characteristics, and reflects state-anxiety proneness. State anxiety is associated with activation or arousal of the autonomic nervous system. State and trait anxiety refers to two related but quite different constructs. Whereas state anxiety has been already hypothesized to enhance responses to vestibular stimulation, no consistent correlation between trait anxiety and vestibular nystagmus has yet been exhibited (Yardley et al 1995). There is no evidence as to whether trait anxiety and vestibular function might be related. 
Table 1. Group Characteristics

\begin{tabular}{|c|c|c|c|c|c|c|c|c|c|c|c|}
\hline & Female $n$ & $\begin{array}{c}\text { Age } \\
\text { (years) }\end{array}$ & $\begin{array}{l}\text { Education } \\
\text { (years) }\end{array}$ & Trait anxiety & $\mathrm{CESD}^{b}$ & Internality $^{c}$ & Power other ${ }^{c}$ & Chance $^{c}$ & $\begin{array}{l}\text { Right-left } \\
\text { orientation }\end{array}$ & $\mathrm{GEFT}^{d}$ & $\begin{array}{c}\text { Spatial } \\
\text { orientation }\end{array}$ \\
\hline \multicolumn{12}{|l|}{ HTA } \\
\hline $\begin{array}{l}\text { Group } \\
(n=9)\end{array}$ & 4 & & & & & & & & & & \\
\hline Mean & & 20.8 & 13.7 & $55.3^{a}$ & $23.7^{a}$ & 28.6 & $13.7^{a}$ & 20.2 & 15 & 12.7 & 33.6 \\
\hline SD & & 1.5 & 0.7 & 4.2 & 8.0 & 4.5 & 4.8 & 3.9 & 4.5 & 5.2 & 11.7 \\
\hline \multicolumn{12}{|l|}{ LTA } \\
\hline $\begin{array}{l}\text { Group } \\
(n=11)\end{array}$ & 7 & & & & & & & & & & \\
\hline Mean & & 20.2 & 13.4 & $27.1^{a}$ & $5.3^{a}$ & 34.5 & $7.8^{a}$ & 15.8 & 16.1 & 14.6 & 33.1 \\
\hline $\mathrm{SD}$ & & 1.4 & 0.7 & 4.8 & 1.5 & 6.3 & 1.9 & 4.5 & 3.1 & 3.3 & 11.7 \\
\hline
\end{tabular}

In the present experiment, we thought it was necessary to study not only VOR gain, but also an additional parameter: the shift of the beating field. This shift is a measure of the extent that the nystagmus "envelope" drifts from the primary eye position (Frenzel 1928), and represents the eye deviation. Mishkin and Melvill Jones (1966) suggested that this drift, originating from the fast phase of the nystagmus, might have the function of turning the eye quickly in the direction of head orientation so that the eye could pick up an intended point of visual interest more quickly. Since then, several authors have claimed that this parameter participates in an orientation strategy (Barnes 1976; Barnes and Forbat 1979; Borel et al 1994; Chun and Robinson 1978; Schmid and Zambarbieri 1988; Siegler et al 1998). By studying not only the VOR gain but also the shift of the beating field, we wanted to examine whether a different strategy of orientation at the oculomotor level would be exhibited in anxiety. This strategy would be dependent on the multisynaptic pathway involving a network of brainstem neurons that generate repositioning saccades in the direction of head movement.

\section{Methods and Materials}

\section{Subjects}

Twenty individuals (11 women, 9 men, mean age 20.5, SD 1.5; see Table 1) were selected from a pool of 253 undergraduate students. These selected volunteers were recruited on the basis of their extreme trait anxiety score as assessed by the State-Trait Anxiety Inventory (Spielberger et al 1983). Two groups of participants were formed, according to their low $(<31)$ or high $(>50)$ scores on the trait anxiety scale of the STAI. The scores of 31 and 50 represent the upper and lower 15 percentile, respectively, for the 253 screened students. In these 2 groups, subjects reporting any history of neurological disease or vestibular impairment, or who were currently under medical treatment were excluded from the study. All selected subjects were right-handed. We thus conducted the experiment with 1 group of 11 (Low Trait Anxiety group LTA) and one of 9 (High Trait Anxiety group HTA) participants.

Table 1 lists the characteristics for each group of subjects and shows the responses of subjects on questionnaire measures. The responses to self-report psychometric inventories and questionnaires revealed differences among the groups that were consistent with their group definitions. No difference was found for the other responses.

Subjects gave their written informed consent to the study after the procedure had been fully explained and were paid for their time. They were cautioned to maintain normal sleeping hours and to abstain from alcohol the day before the experiment. On the day of the experiment, all of the subjects reported they complied with the request for normal sleeping hours and alcohol abstinence. None of them had participated in this kind of experiment before.

\section{Questionnaires and Interview Measures}

The initial screening of subjects was composed of the State-Trait Anxiety Inventory, the Center for Epidemiologic Studies Depression scale (Radloff 1977), and the Internality, Powerful others and Chance scales (Levenson 1981). A psychologist supervised by a faculty psychiatrist administered the interview of the eligible students for the study, that was based on the Mini International Neuropsychiatric Interview (Lecrubier et al 1997). Six of the 9 students from the HTA group described the presence of some characteristics listed in the DSM-IV for the following anxiety disorders: agoraphobia without history of panic disorder, general anxiety disorder (2 subjects), social phobia (2 subjects) and actual panic disorder (2 subjects). Subjects from the LTA group had no history of anxiety disorder nor did they meet the DSM-IV criteria for any psychiatric disorder.

Immediately before the start and immediately after completion of the experiment, participants filled out the state scale of STAI. The score on the state anxiety scale was used for a preand postexperiment assessment of the participants' level of anxiety. 


\section{Experimental Setup}

Subjects were seated on a remote-controlled mobile robot (Robuter, Robosoft, France) that performed rotations about the Earth-vertical axis (see Ivanenko et al 1997 for details of the experimental setup). The robot's motion (speed, position) could be controlled by either a remote computer via wireless modems (to generate passive rotations of the subject), or by the subjects themselves by means of a joystick. Horizontal eye movements (eye rotations around the longitudinal body axis) were measured throughout the experiment with an infrared system (IRIS, Skalar). Eye movements and robot rotation were recorded at sampling rate of $100 \mathrm{~Hz}$. Subjects were seated with the head fixed at the center of rotation by two soft cushions mounted on the robot, and wore headphones delivering wide band noise to prevent auditory spatial cues.

\section{Experimental Procedure}

In each trial, subjects were first submitted to a passive wholebody rotation (stimulus) in darkness and then had to reproduce, in the same direction, the stimulus angular magnitude by driving the robot with the joystick (response), while still in the dark (Siegler et al 1998). Stimulus angles were $80^{\circ}, 167^{\circ}$, and $340^{\circ}$ and were imposed with triangular velocity profiles, with peak velocities ranging from 30 to $60 \%$ s. Subjects performed a total of 12 trials ( 6 clockwise, 6 counterclockwise). We asked subjects to keep their eyes open and to look "far away" in front of them during stimulus and response, to prevent them, as much as possible, from imagining a head-fixed target.

\section{Eye Movement Analysis}

The gain of the vestibulo-ocular reflex (VOR) was computed in the following way: for each slow phase, polynomials of degree 3 were fitted to the recorded data of eye and robot angular position to minimize the mean square error between the fitted curve and the data. The regression polynomials were differentiated to yield an approximation of angular eye velocity and of robot velocity during each slow phase. The VOR gain during each slow phase was then calculated by averaging the ratio of eye velocity to robot velocity across the duration of the slow phase. To avoid aberrant values of VOR gain, slow phases that occurred while the robot's angular velocity was under $10 \%$ s were omitted.

In addition to the VOR gain, we studied the shift of the beating field of ocular nystagmus. To do so, we measured, for each rotation, the maximum value of this shift (see Siegler et al 1998 for details). For convenience, we will refer to this value as "gaze shift." Gaze shift was taken as positive when the shift was in the quick phase direction (anticompensatory) and negative in the slow phase direction (compensatory), so that we could pool data from clockwise and counterclockwise rotations.

\section{Statistical Analysis}

The effect of group on anxiety levels was evaluated using analysis of variance (ANOVA) with repeated measures. The effect of group on VOR gain was analyzed by one-way ANOVA, as well as the effect of group on gaze shift. State anxiety was used as a covariable in a repeated measures analysis of covariance that compared HTA and LTA groups on the gaze shift during stimulus and reproduction.

\section{Results}

\section{Anxiety Levels}

The state anxiety of the participants was assessed using the state portion of the Spielberger's STAI, before and immediately after the experiment. An analysis of variance (ANOVA) with the between subjects factor "group" (high or low trait anxiety) and the within subject factor "period" (pre, post) was performed on the obtained state anxiety scores. Significant main effects were found for the factors Group $(F(1,18)=14.44, p<.001)$ and Period $(F$ $(1,18)=10.40, p<.005)$. A Newman-Keuls post hoc analysis revealed that, whereas the High Trait Anxiety (HTA) group exhibited a significant decrease in state anxiety between pre- and posttesting $(p<.006)$, the Low Trait Anxiety (LTA) group showed a non-significant decrease (pretesting, HTA: Mean $\pm \mathrm{SD}=38.9 \pm 7.9$, LTA: $28.5 \pm 7.2$; posttesting, HTA: $30.9 \pm 4.2$; LTA: $24.8 \pm 5.2$ ).

\section{Reproduction Task}

To examine subjects' performance on the reproduction task, we computed the amplitude ratio of the reproduction angle to stimulus angle. A ratio of 1 reflects a perfect fit between stimulus and reproduction. Overall mean values of amplitude ratio were $1.00 \pm 0.24$ in LTA group and $1.05 \pm 0.22$ in HTA group, indicating accurate reproduction for both groups.

\section{Eye Movements}

The relative small amplitude of stimulus angles used in the present experiment did not always induce strong perrotatory nystagmus. Eye recordings were visually inspected and only trials exhibiting prominent per-rotatory nystagmus during both stimulus and reproduction rotations were kept for analysis. Eye recordings should exhibit at least two clearly distinct slow phases, during both stimulus and reproduction, to fit the criteria of selection. Of our recordings, $62 \%$ in the LTA group and $51 \%$ in the HTA group were kept for analysis.

Eye movements were characterized by the VOR gain and the gaze shift during vestibular nystagmus, i.e., during both stimulus and reproduction rotations. During stimulus rotations, mean VOR gain was $0.55 \pm 0.23$ in the LTA group and $0.54 \pm 0.24$ in the HTA group. During reproduction rotations, those means were down to $0.44 \pm$ 0.27 and $0.45 \pm 0.23$, respectively. Therefore, comparisons between the HTA and LTA groups did not reveal any 
A Low Trait Anxious subject

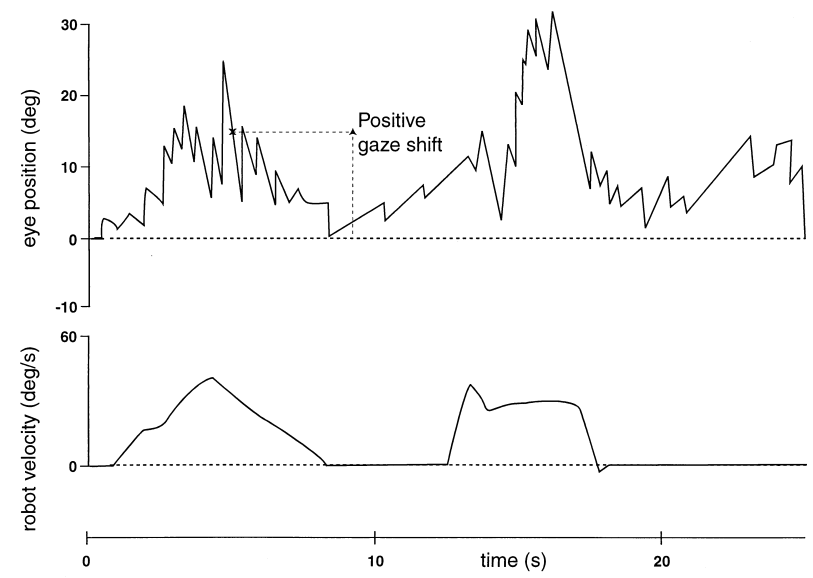

B High Trait Anxious subject
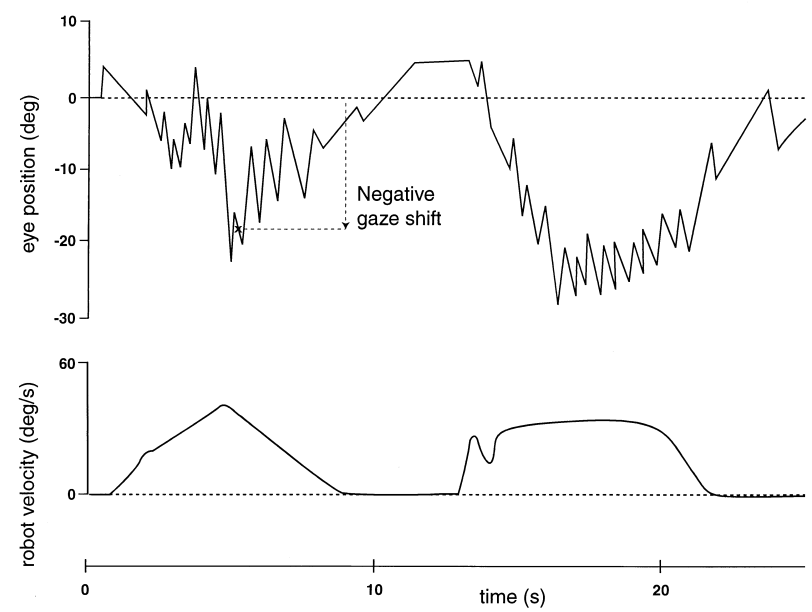

Figure 1. Sample trials with (A) a positive gaze shift in a Low Trait Anxious subject and with (B) a negative gaze shift in a High Trait Anxious subject. The positive gaze shift indicates an anticipatory orientation strategy, linked to the use of an allocentric frame of reference. The negative gaze shift, representing a lag, could be consistent with an egocentric strategy. Upper trace: eye position. Lower trace: robot angular velocity. In each, stimulus rotation within the first 10 seconds is followed by a brief pause and then reproduction of the movement.

significant differences in VOR gain values. There was no consistent pattern of correlation between state scores and nystagmus induced by rotations. Furthermore, there was a significant intra-individual correlation between VOR gain during stimulus and during reproduction $(r=.58, p<$ $.0001, n=111$ ).

A significant discrepancy in the mean gaze shift was observed between the LTA and HTA group (Figure 1), during stimulus presentation (LTA: mean $=9.76 \pm 9.00^{\circ}$, HTA: $1.28 \pm 15.13^{\circ}$; one-way breaking ANOVA, $F$ $[1,107]=14.17, p<.001)$ as well as during reproduction $\left(\right.$ LTA: mean $=10.27 \pm 12.61^{\circ}$, HTA: $3.53 \pm 15.41^{\circ}$; one-way breaking ANOVA, $F[1,108]=6.23, p=.02$ ), although subjects in both groups performed reproduction rotations with the same accuracy. In fact, the small mean values of gaze shift observed in the HTA group were due to a larger number of negative shifts than in the LTA group. There was no significant difference in absolute values of gaze shift during both stimulus and reproduction rotations between the LTA group (mean $=13.16 \pm 7.87^{\circ}$, and $13.94 \pm 9.54^{\circ}$, respectively) and the HTA group $\left(\right.$ mean $=13.34 \pm 6.87^{\circ}$ and $13.64 \pm 7.71^{\circ}$, respectively). A 2-way analysis of variance with repeated measures on one factor (gaze shift during stimulus and during reproduction) and with one independent factor (group) and one covariable (score on the state portion of the STAI before the experiment) was performed. This test showed a significant main effect of group $(F[1,106]=6.11, p=.01)$, but no interaction, signifying no effect of state anxiety on the difference between groups in gaze shift.

\section{Discussion}

In our study, we compared 2 groups of subjects differing in level of trait anxiety, with a vestibular perception test in darkness. All the subjects managed to perform the task and to reproduce the imposed stimulus with relative accuracy. The eye movements recordings allowed us to study several parameters. Gaze shift exhibited a dramatic difference in the 2 groups, whereas the VOR gains were comparable. In the following discussion we consider briefly the lack of differences in VOR gain and comment these results in light of other findings concerning this more commonly studied parameter. We then address more extensively the novel findings of gaze shift differences and discuss how this phenomenon might relate to self-orienting behavior.

It has previously been reported that state anxiety is correlated with an enhanced gain of the VOR (Yardley et al 1995). In our study, we did not observe such an effect. Yet, it is important to note that in our case, the experimental situation was not anxiogenic: our subjects exhibited a decrease in their anxiety from the beginning of the experiment to the end. Furthermore, mental alertness did not differ between the subjects, because all of them attended very well to the stimulus, as indicated by the accuracy of their reproduction.

We did not find any interaction between trait anxiety level and VOR gain. This can be compared to the first experiment of the findings of Yardley et al (1995), that did not exhibit any significant difference in nystagmus characteristics between groups of agoraphobic individuals and of normal controls. They found that fear-rating scores completed before testing were correlated with an enhanced VOR gain during caloric stimulation. In another experiment of the same study, Yardley et al found a significant 
effect of induced mental stress on the maximum slow phase velocity during impulsive rotation testing. These results combined with our present study seem to emphasize the role of state anxiety on the usual VOR parameters. Inducing stressful mental activity is a way of experimentally manipulating levels of state anxiety; caloric stimulation can be considered as an anxiogenic testing as well. Because our experimental manipulation did not have any effect on state anxiety, it is not surprising that there is no interaction between state or trait anxiety and VOR gain.

The most intriguing result of the present study is the difference observed in the gaze shift between LTA and HTA groups. The question of the functional role of this shift needs to be addressed. The shift of the beating field is induced by the fast phases of nystagmus that do not simply return the eye to the center of the orbit, but also direct the eye beyond this primary position in the direction of head movement (anticompensatory direction). Indeed, during active or passive head movements in the dark, the saccadic system seems to be triggered by the head movement so as to quickly orient the gaze in the direction of head motion (Schmid and Zambarbieri 1998). In other words, the nystagmus fast phases allow the eyes to anticipate head movements, thus facilitating rapid gaze movements toward a point of interest in the environment (Barnes and Forbat 1979; Chun and Robinson 1978; Melvill Jones 1964; Zambarbieri et al 1997).

The saccadic system has also been considered as a mechanism implicated in orienting processes (Borel et al 1994). Jürgens and Mergner (1989) suggested that the fast phases of vestibular nystagmus are modulated by highlevel centers that process sensory information. It has also been shown that the VOR is under the control of cortical representations of the vestibularly perceived self-rotation and target spatial localization (Berthoz 1989; Bloomberg et al 1991; Collewijn 1989).

The shift of the beating field has been recently studied in humans (Siegler et al 1998) and proposed to be related to the orientation strategy of the subject. In this study, the authors investigated whether the gaze shift observed during vestibular stimulation corresponded to an orienting behavior, by submitting the subjects to two conditions. In the first condition, subjects were not shown the darkened experimental room and were asked to pay attention exclusively to their sensations of body rotations in place. In the second, they were shown the room before each trial and were asked to refer their perceived movements to external landmarks. Based on their results, the authors proposed that different strategies could be used to solve an orienting task. Small shifts of the beating field were exhibited when subjects had to use an egocentric frame and did not refer to any external center of interest in the room. On the contrary, large shifts were exhibited when subjects were asked to use an internal representation of the room, i.e., an allocentric frame of reference. Therefore, the shift of the beating field could participate in the anticipation strategy. Anticipatory space-oriented reactions have been suggested to belong to the repertoire of human navigation behavior (Grasso et al 1998). Anticipatory orientation would allow the achievement of a stable frame of reference in time aimed at anticipating future gaze direction and motor events. Because such anticipation could be linked to an allocentric strategy, a lag would be consistent with an egocentric strategy, because the absence of space representation would lead to a smaller or negative eye deviation.

In the current study, the HTA group exhibited more negative shifts than the LTA group. It is therefore possible that HTA subjects use a different strategy than LTA subjects. For instance, High trait anxiety subjects may use a mode of spatial processing based on an egocentric frame of reference, whereas low trait anxiety subjects would more easily use an allocentric frame of reference. If anxious trait subjects indeed do not use an allocentric frame of reference, it may be because they do not pay attention to their surroundings. In fact, some psychological and functional evidence point to theories linking oculomotor control and attention (Büchel and Friston 1997; Büchel et al 1998; Kowler et al 1995; Kustov and Robinson 1996; Rizzolatti et al 1987; Sheliga et al 1994). It has for example been demonstrated that perceptual attention determines the endpoint of a saccade (Kowler et al 1995). It is now demonstrated that attention and eye movement processes share functional anatomical areas in the human brain (Corbetta 1998).

Several studies point to the role of attentional strategies in anxiety (Ahles et al 1983; Leventhal et al 1979; Sartory et al 1982; Watts 1974). Regarding clinical observation, it is known that phobic and agoraphobic patients, when their level of anxiety is high, are inclined to reduce attention to the perceptual field and external environment (Watts 1989). Watts suggested that this kind of avoidance is probably an involuntary response to experiencing excessive anxiety, and is similar in this respect to the impulse to escape physically from the anxiety-arousing situation. Although our study did not produce any stress in the subjects, the negative shift observed in high trait anxious subjects could provide an observable cue that perceptual avoidance is taking place.

Evidence of dysfunction of spatial orientation found in agoraphobia (Jones et al 1996; Kallai et al 1995) may point to an alternative to the attentional hypothesis to explain possible deficits in allocentric orienting behavior. During navigation in a complex environment, panic agoraphobic patients got lost more often and utilized fewer navigation points. The maps drawn afterwards by these 
patients were inaccurate (Kallai et al 1995). The pattern of results in panic agoraphobic patients is consistent with the negative shifts (interpreted as an egocentric orienting behavior) observed in our group of anxious subjects. It is known that the hippocampus participates in the encoding of the context of information and in allocentric memory (see Metcalfe and Jacobs 1998 for review). Intense stress releases corticosterone, that interferes with the normal functions of the hippocampus (McEwen et al 1992). The lack of use of an allocentric frame of reference could then be viewed as an expression of the cognitive deficits induced by corticosteroids (see Lupien and McEwen 1997 for a review on the effects of corticosteroids on cognition).

To summarize our results, we have observed specific differences in vestibular driven eye movements between subjects exhibiting low and high trait anxiety, and have proposed to interpret this oculomotor behavior in terms of differing strategies for self-orientation. The eye deviation exhibited by HTA subjects and the hypothesis of an egocentric orienting strategy for these subjects are in line with observations about agoraphobic behavior, and could reflect consequences of attentional mechanisms. Nevertheless, biological explanations as to the role of corticosteroids should also be considered. Further investigation into the potential link between the beating field of vestibular nystagmus and the development of agoraphobia may reveal a physiological manifestation of this so-called cognitive disorder.

Supported by SmithKline Beecham FRM and GIS-Sciences de la Cognition. We thank Joe McIntyre for his helpful comments on the manuscript, as well as France Maloumian for technical assistance.

\section{References}

Ahles TA, Blanchard EB, Leventhal H (1983): Cognitive control of pain: attention to the sensory aspects of the cold pressure stimulus. Cognit Ther Res 7:159-178.

Barnes GR (1976): The role of the vestibulo-ocular reflex in visual target acquisition. J Physiol (London) 258:64-65.

Barnes GR, Forbat LN (1979): Cervical and vestibular afferent control of oculomotor response in man. Acta Otolaryngol 88:79-87.

Berthoz A (1989): Coopération et substitution entre le système saccadique et les "réflexes" d'origine vestibulaire: faut-il réviser la notion de "réflexe"? Revue de Neurologie (Paris) 145:513-526.

Bloomberg J, Melvill Jones G, Segal BN (1991): Adaptive modification of vestibularly perceived rotation. Exp Brain Res 84:47-56.

Borel L, Le Goff B, Charade O, Berthoz A (1994): Gaze strategies during linear motion in head-free humans. $\mathrm{J} \mathrm{Neu}$ rophysiol 5:2451-2466.

Büchel C, Friston KJ (1997): Modulation of connectivity in visual pathways by attention: cortical interactions evaluated with structural equation modelling and fMRI. Cereb Cortex 7:768-778.

Büchel C, Josephs O, Rees G, Turner R, Frith CD, Friston KJ (1998): The functional anatomy of attention to visual motion. A functional MRI study. Brain 121:1281-1294.

Chun KS, Robinson DA (1978): A model of quick phase generation in the vestibulo-ocular reflex. Biol Cybern 28: 209-221.

Clark DB, Hirsch BE, Smith MG, Furman JMR, Jacob RG (1994): Panic in otolaryngology patients presenting with dizziness or hearing loss. Am J Psychiatry 151:1223-1225.

Collewijn H (1989): The vestibulo-ocular reflex: an outdated concept. Prog Brain Res 80:197-209.

Corbetta M (1998): A common network of functional areas for attention and eye movements. Neuron 21:761-773.

Frenzel H (1928): Rucknystagmus als Halsreflex und Schlagfeldverlagerung des labyrinthaeren Drehnystagmus durch Halsreflex. Z Hals Nasen Ohrenheilkd 147:177-187.

Grasso R, Prevost P, Ivanenko YP, Berthoz A (1998): Eye-head coordination for the steering of locomotion in humans: an anticipatory synergy. Neurosci Lett 253:115-118.

Hoffman DL, O’Leary DP, Munjack DJ (1994): Autorotation test abnormalities of the horizontal and vertical vestibulo-ocular reflexes in panic disorder. Otolaryngol Head Neck Surg 110:259-269.

Ivanenko YP, Grasso R, Israël I, Berthoz A (1997): The contribution of otoliths and semicircular canals to the perception of two-dimensional passive whole-body motion in humans. J Physiol 502:223-233.

Jacob RG, Furman JMR, Durrant JD, Turner SM (1996): Panic, agoraphobia, and vestibular dysfunction. Am J Psychiatry 153:503-512.

Jacob RG, Moeller MB, Turner SM, Wall C (1985): Otoneurological examination in panic disorder and agoraphobia with panic attacks: a pilot study. Am J Psychiatry 142:715-720.

Jones RB, Humphris G, Lewis T (1996): Do agoraphobics interpret the environment in large shops and supermarkets differently? Br J Clin Psych 35:635-637.

Jürgens R, Mergner T (1989): Interaction between cervico-ocular and vestibulo-ocular reflexes in normal adults. Exp Brain Res 77:381-390.

Kallai J, Koczan G, Szabo I, Molnar P, Varga J (1995): An experimental study to operationally define and measure spatial orientation in panic agoraphobic subjects, generalized anxiety and healthy control groups. Behav Cogn Psychotherapy 23:145-152.

Kowler E, Anderson E, Dosher B, Blaser E (1995): The role of attention in the programming of saccades. Vision Res 35: 1897-1916.

Kustov AA, Robinson DL (1996): Shared neural control of attentional shifts and eye movements. Nature 384:74-77.

Lecrubier Y, Sheehan D, Weiller E, Amorim P, Bonora LI, Harnett K, et al (1997): The Mini International Neuropsychiatric Interview: reliability and validity according to the CIDI. Eur Psychiatry 12:224-231.

Levenson H (1981): Differentiating among internality, powerful others and chances. In: Lefcourt H, editor. Research with the Locus of Control Construct, Vol. I. Assessment Methods. New York: Academic Press, 15-63. 
Leventhal H, Brown D, Shacham S, Engquist G (1979): Effect of preparatory information about sensations, threat of pain and attention on cold pressor distress. J Pers Soc Psychol 37:688 714.

Lupien SJ, McEwen BS (1997): The acute effects of corticosteroids on cognition: integration of animal and human model studies. Brain Res Brain Res Rev 24:1-27.

McEwen BS, Gould EA, Sakai RR (1992): The vulnerability of the hippocampus to protective and destructive effects of glucocorticoids in relation to stress. Br J Psychiatry 15:1823.

Melvill Jones G (1964): Predominance of anti-compensatory oculomotor response during rapid head rotation. Aviat Space Environ Med 35:965-968.

Metcalfe J, Jacobs WJ (1998): Emotional memory: The effect of stress on "cool" and "hot" memory systems. Psychol Learning Motivation, 38:187-222.

Mishkin S, Melvill Jones G (1966): Predominant direction of gaze during slow head rotation. Aerospace Med 37:897-900.

Radloff LS (1977): The CES-D scale: a self report depression scale for research in the general population. Appl Psychol Measure 1:385-401.

Rizzolatti G, Riggio L, Dascola I, Umilta C (1987): Reorienting attention across the horizontal and vertical meridians: evidence in favor of a premotor theory of attention. Neuropsychologia 25:31-40.

Sartory G, Rachman S, Grey SJ (1982): Return of fear: the role of rehearsal. Behav Res Ther 20:123-133.

Schilder P (1933): The vestibular apparatus in neurosis and psychosis. J Nerv Ment Dis 78:1-23.

Schmid R, Zambarbieri D (1988): Vestibular nystagmus as a manifestation of a strategy of head-eye coordination. In: Lüer
G, Lass U, Hoffman J, editors. Eye Movement Research Physiological and Psychological Aspects. Zurich: CJ Hogrefe, 146-170.

Sheliga BM, Riggio L, Rizzolatti G (1994): Orienting of attention and eye movements. Exp Brain Res 98:504-522.

Siegler I, Israël I, Berthoz A (1998): Shift of the beating field of vestibular nystagmus: an orientation strategy? Neurosci Lett 254:93-96.

Sklare DA, Stein MB, Pikus AM, Uhde TW (1990): Dysequilibrium and audiovestibular function in panic disorder: symptom profiles and test findings. Am J Otology 11:338-341.

Spielberger CD, Gorsuch RL, Lushene R, Vagg PR, Jacobs GA (1983): Manual for the State-Trait Anxiety Inventory (STAI). Palo Alto: Consulting Psychologists Press.

Watts FN (1974): The control of spontaneous recovery of anxiety in imaginal desensitization. Behav Res Ther 12:57-59.

Watts FN (1989): Attentional strategies and agoraphobic anxiety. Behav Psychotherapy 17:15-26.

Witkin HA, Oltman PK, Karp SA (1971): A Manual for the Embedded Figure Test. Palo Alto: Consulting Psychologists Press.

Yardley J, Watson S, Britton J, Lear S, Bird J (1995): Effects of anxiety arousal and mental stress on the vestibulo-ocular reflex. Acta Otolaryngol 115:597-602.

Yardley L, Burgneay J, Nazareth I, Luxon L (1998): Neurootological and psychiatric abnormalities in a community sample of people with dizziness: a blind, controlled investigation. J Neurol Neurosurg Psychiatry 65:679-684.

Zambarbieri D, Schmid R, Versino M, Beltrami G (1997): Eye-head coordination toward auditory and visual targets in humans. J Vest Res 7:251-263. 